\title{
ROLE OF EFFECTIVE PROJECT MANAGEMENT IN REDUCING DRUG DEVELOPMENT COST
}

\author{
FAISAL BA ZAIDI ${ }^{1 *}$, SALMA AHMED ${ }^{1}$, MUNISH MAKKAD ${ }^{2}$
}

${ }^{1}$ Department of Business Administration, Faculty of Management Studies and Research, Aligarh Muslim University, Aligarh, Uttar Pradesh, India. ${ }^{2}$ Department of Management, Birla Institute of Technology, Noida, Uttar Pradesh, India.

Email: faisalbazaidi@gmail.com

Received: 23 November 2016, Revised and Accepted: 30 November 2016

ABSTRACT

Pharmaceuticals are passing through the difficult phase due to increasing numbers of patents expiry along with increasing cost of drug development. Protocol design, regulatory cycle time, site selection, patient enrollment and monitoring are some of the cost contributing elements for late phase clinical trials. This paper applies the principles of project management and suggests means to reduce the cost of late phase drug development. It also throws light on the critical role that a project manager can play in overall drug development process.

Keywords: Cost of drug development, Effective project management, Reduction in cost of drug development.

(c) 2017 The Authors. Published by Innovare Academic Sciences Pvt Ltd. This is an open access article under the CC BY license (http://creativecommons. org/licenses/by/4. 0/) DOI: http://dx.doi.org/10.22159/ajpcr.2017.v10i3.16186

\section{INTRODUCTION}

Drug development is a complicated process and extremely challenging to execute. It takes lots of efforts and expertise to develop one drug and it is estimated that one drug reaches to the market after around 1000 failed discoveries [1]. The cost of a typical drug development is around 1.2 billion USD [2] and it is increasing each year while more number of failures at later stage makes it costlier. Data suggest that only one out of 25 preclinical molecules would reach to the approval status [3] while the success rate of Phase I to approval is suggested as $12 \%$ [4]. The success of clinical trial also varies with phases and it is reported as $67 \%, 41 \%$ and $55 \%$ for Phase I, Phase II and Phase III trials, respectively [1]. Two separate studies from year 2010 to year 2011 suggest the clinical trial success probabilities as 19\% and 9\% [1] separately.

Pharmaceutical organizations are passing through difficult phase due to increasing numbers of patent expiry of their marketed products which in turn impact their revenue. It is estimated that sales of major pharmaceutical companies may have been reduced to $44 \%$ because of patent expiry between years 2009 and 2014 with brands worth USD 128 billion losing the patent in the same time period [2].

On the other hand, it has become extremely difficult to launch new drug due to increasing development cost. There is no doubt that late phase development, i.e. Phase II and Phase III are the costliest phases of drug development and constitute around $90 \%$ of the total development cost [2]. In the absence of blockbuster and first in class molecule, it is becoming more and more demanding for pharmaceutical companies to get their investment back and one report estimated that only one out of five drugs will be able to provide the revenue equivalent to or more than its investment [2]. It has become a grim assignment for pharmaceutical companies to persuade the investors and partners to invest in late phase development in the absence of not so promising result from earlier phases. It is very challenging to forecast the budget of a clinical trial, and only $14 \%$ financial planners will be sure of their budget [5].

Industry experts estimated that late phase drug development is the costliest phase of drug development. Costly late phase development can be considered as one of the reasons that more than $40 \%$ of the marketed products during 2003-2013 were acquired [6] as pharmaceutical companies would prefer to invest in the molecule which is in later phase to avoid major loses. If one looks closely at the activities performed in the late phase drug development and would know that these are majorly project management activities. These activities would include protocol design, vendor identification and selection, site identification and selection, regulatory submission and approval, site initiation, patient's enrollment; site monitoring, data management, audit/inspection and site close out, etc. Fig. 1 illustrates that each of these activity is part of a project or a complete project in itself depending on the size of the trial, e.g., for a large Phase III trial, site selection can be a project in itself while in case of relatively smaller Phase II trial, it can be one of the tasks of overall project. The focus below is on those activities which are major cause of cost increase and can be managed through effective project management.

\section{PROTOCOL DESIGN}

Clinical trial protocol increasingly getting complicated day by day due to various reasons. Increasing knowledge of science, i.e., availability of disease history, demography, etiology, molecular knowledge of the drug, and biomarker studies, are some of the reasons for increasing complexities of the clinical trial. Increasing size of the trials is another major factor for the complexities of the trial as each geographical region will have its own concern and the data coming from each region would require to be addressed. Some countries do not allow placebo control studies and it is one of the important considerations during protocol designing while planning the trial which involves such regions. On the other hand, protocol complexities would also impact various other associated activities such as patient enrollment, drug shipment, and monitoring which certainly would influence the cost of clinical trial. A survey conducted in year 2011 with 79 organizations involved in conducting clinical trial revealed that trial design was the first factor in determining the frequency of the monitoring in the study [7]. Another report suggests that total procedures per trial protocol have increased from 105 in year 2000-2003 to 166 in year 2008-2011 [8], while clinical trial treatment period (median days) increased from 140 to $175[4]$.

It is noticed that protocol writing team work independently and inputs are sought from other functions only in the later phase of protocol development. It is important that collaboration in the beginning itself is very significant to discuss the upcoming challenges of proposed protocol 


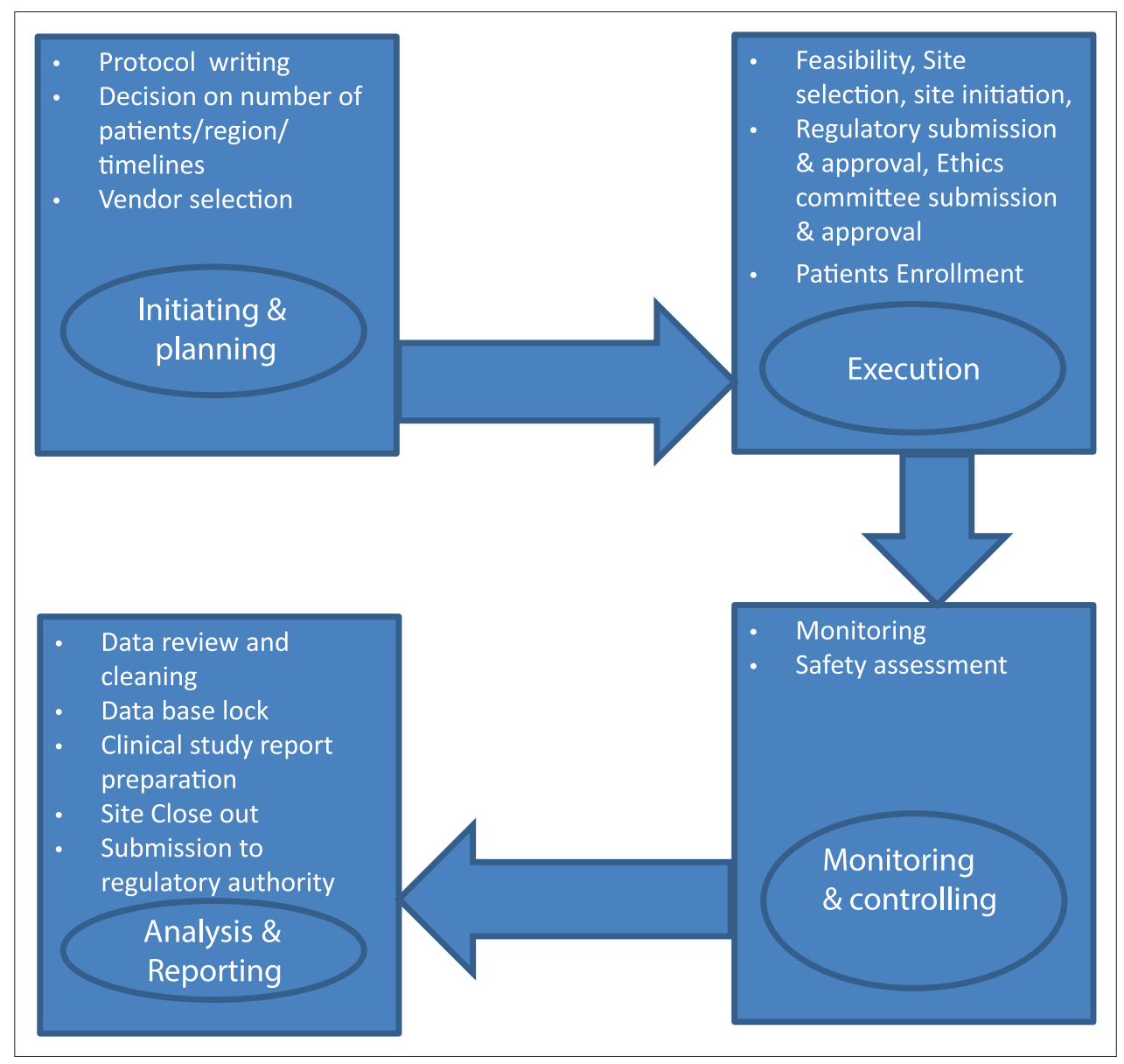

Fig. 1: Typical activities of clinical trial and phases of project management

and inputs from regulatory, clinical operations, data management, manufacturing, and various other functions. It would be a help for medical writer to understand the anticipated challenges and get the solutions from other team members, for instance, the manufacturing unit may not be equipped to provide the sufficient investigation product as per anticipated high patient enrollment rate, clinical operation may not be having sufficient resources to monitor the data generated at sites, etc. It is interesting to note here that $43 \%$ of the protocol amendments happened even before first patient enrollment [1]. It is understandable that it is difficult to simplify a complex trial protocol for various scientific reasons; however, the associated cost can certainly be reduced by improving the planning at protocol development. Complexity of the protocol may lead to increase in clinical trial duration by $64 \%$ [9]. There is software available to help in deciding the number of patients, regions, etc. which can help in cost saving by better planning [10] as approximately $45 \%$ of the pharmaceutical financial professionals would fail to forecast the budget accurately [10].

\section{REGULATORY SUBMISSION AND APPROVAL}

One of the most important milestones for conducting clinical trial is to get a go ahead from regularity authorities. It requires lots of expertise to avoid any delay and queries from regulatory authorities as delay in getting regularity approval may impact the overall conduct and planning of a clinical trial, e.g., delay in getting approval for an asthmatic or allergic rhinitis trial may postpone the overall enrollment of the patients in the trial. It is interesting to note that a single day delay of market authorization approval can lead to a potential sales loss of USD 8 million [11] It is very difficult to forecast the regulatory approval time for the multicenter trial because of varied timeline and dynamic structure of different regulatory authorities. However, planning may help in saving some time like getting feedback from the key opinion leaders and clinical practitioners during the protocol designing would surely help in avoiding queries from regulatory authorities on conduct and design of the study. There are some regulatory authorities which allow to have a meeting to discuss the design and such opportunities could be utilized to avoid queries later. According to a study conducted on 302 first new molecular entities drug applications first submitted to the Food and Drug Administration (FDA) for the period between years 2000 and years 2012, it was found that 71 applications failed to get approval in the first submission and required at least second submission which added a medial delay of 435 days [12]. Time is precious when it comes to drug development as even single day delay may cost a lot to the pharmaceutical companies. There is an increase of $11 \%$ reported in the regulatory cycle time between years 2000 and years 2005 though there is decrease in US FDA regulatory approval time reported in recent past [1]. Sometime it also happen that outcome of a Phase II trial would lead to next phase. According to a study which covered over 3600 protocol, over one-third of the protocol amendments would have been avoided by better planning in the beginning. Each protocol amendment directly impacts the cost as it may be associated with other process and procedure, i.e., principle investigator's fee and site fee, clinical procedure, new vendors, additional drug and other supply at sites, change in informed consent and other patient material as it would also require the ethics committee approval and around $69 \%$ of the protocol would have at least one amendment [8]. The same study further added that the cost of each protocol amendment stands as $\$ 453,932$ [1] which is considerable while it should be noted that $37 \%$ of protocol amendments can be avoided thus leading to cost saving [8].

\section{SITE SELECTION AND ENROLLMENT}

Investigator site selection is one of the most challenging tasks in late phase clinical trials as it is increasingly becoming difficult to identify 
newer sites for the trial considering that number of experienced principal investigators is reducing at a rate of $6 \%$ after 2001 [13] which is compelling the pharmaceutical companies to go for first time investigator. On the other hand, a center watch report suggests an alarming dropout rate of $50 \%$ of the first time investigator during the period of years 2001-2004 [13]. Another study for the period of year 2000 and 2007 suggests the reduction of $10 \%$ in the new investigator [14]. There are various reasons for the reduction in number of sites or principle investigators which primarily include the complexity of the trial and increased regulatory vigilance that demands a close oversight, proper attention, accurate documentation and time allocation by the principle investigator and other site staff. It is well understood by the industry that compensation to the principle investigator should be well balanced and they try to keep it as low as possible which in turn results in lack of interest at site level. Site selection contributes to the overall clinical trial cost and USD 20,000-30,000 is the approximate cost of initiating a site while it would cost around USD 1500 to maintain the clinical site [14] which would come enormous for a global clinical trial. This shows that how much cost it would add to the clinical trial if a wrong site is selected. Fig. 2 shows some elements to note during site selections which play a key role to get the best sites selected. Selection of the right site is very important as around $50 \%$ of the site do not meet enrollment target and enroll only one patient or even fail to recruit one patient [14]. A study suggests that site retention costs constitute around $9 \%$ to $16 \%$ of a clinical trial across all phases [1].

Another significant factor which plays a major role in the late phase clinical trial is subject enrollment as it is projected that $80 \%$ trials do not complete the enrollment within timelines [5], and this delay would cost approximately USD 35,000/day [5]. Site performance is also largely dependent on the therapeutic area along with other factors. A data collection study conducted during the year 2006-2010 by Covance suggests that highest non-performing sites come from oncology (60\%) followed by hematology (57\%) while dermatology stands best with just $37 \%$ nonperforming sites [11]. Other factors playing a major role in sites performance are protocol design, placebo arm, trial budget, etc. Subject enrollment is responsible for around $45 \%$ delays in trial completion [14] and around $30 \%$ of phase III clinical trial do not complete on time while this percentage is around $42 \%$ and $31 \%$ for Phase I and Phase II, respectively [11]. According to a study conducted by University of North Carolina in year 2006, around $15-20 \%$ sites would fail to enroll while $30 \%$ of the site would enroll below than estimates [4].

\section{MONITORING}

Monitoring is another cost contributing activity for late phase clinical trial as industry expert estimated around $15-30 \%$ of the cost linked to monitoring [3]. There are various cost which are directly associated with the monitoring, e.g., a protocol amendment would lead to change in the safety or efficacy assessment and informed consent changes which mean additional time spent by the monitor at sites, additional nights spent in hotel and additional flight taken by the monitor. About $100 \%$ source data verification followed by most of the pharmaceutical organizations is also considered as an additional burden by the industry experts as it is not a regulatory requirement. Risk-based monitoring approach is adopted as a replacement of traditional monitoring by some pharmaceutical companies. One of the reports published by Cutting Edge Information suggests that risk-based monitoring can save significant monitoring cost. The report suggests saving of $21 \%$ for Phase III clinical trial and 14\% for Phase II clinical trial conducted by contract research organizations (CROs) while it is around $32 \%$ and $11 \%$ for Phase III and Phase II trials, respectively, in case of trials conducted by the pharmaceutical organization [15]. Cost saving by riskbased monitoring would also depend on therapeutic area, phase, size, protocol complexity of the trial and each study should be evaluated thoroughly before implementing risk-based monitoring. A year 2010 study suggest a cost reduction of $23 \%$ by reducing the source data verification to $50 \%$ and reducing the frequency of monitoring visit from 6 weeks to 10 weeks in an oncology phase 3 study [1]. One of the leading CRO Quintiles suggests a cost reduction of $25 \%$ through risk-based monitoring [16]. Selection and implementation of risk base monitoring data points is very crucial for late phase trials considering that there is $83 \%$ likelihood of receiving market authorization approval from US FDA after completion of Phase III trial [1]

Various other ways of reducing monitoring cost such as preplanned travel booking, corporate tie ups with hotels, assignment of regional monitors to save travel cost would also play a major role in saving monitoring cost.

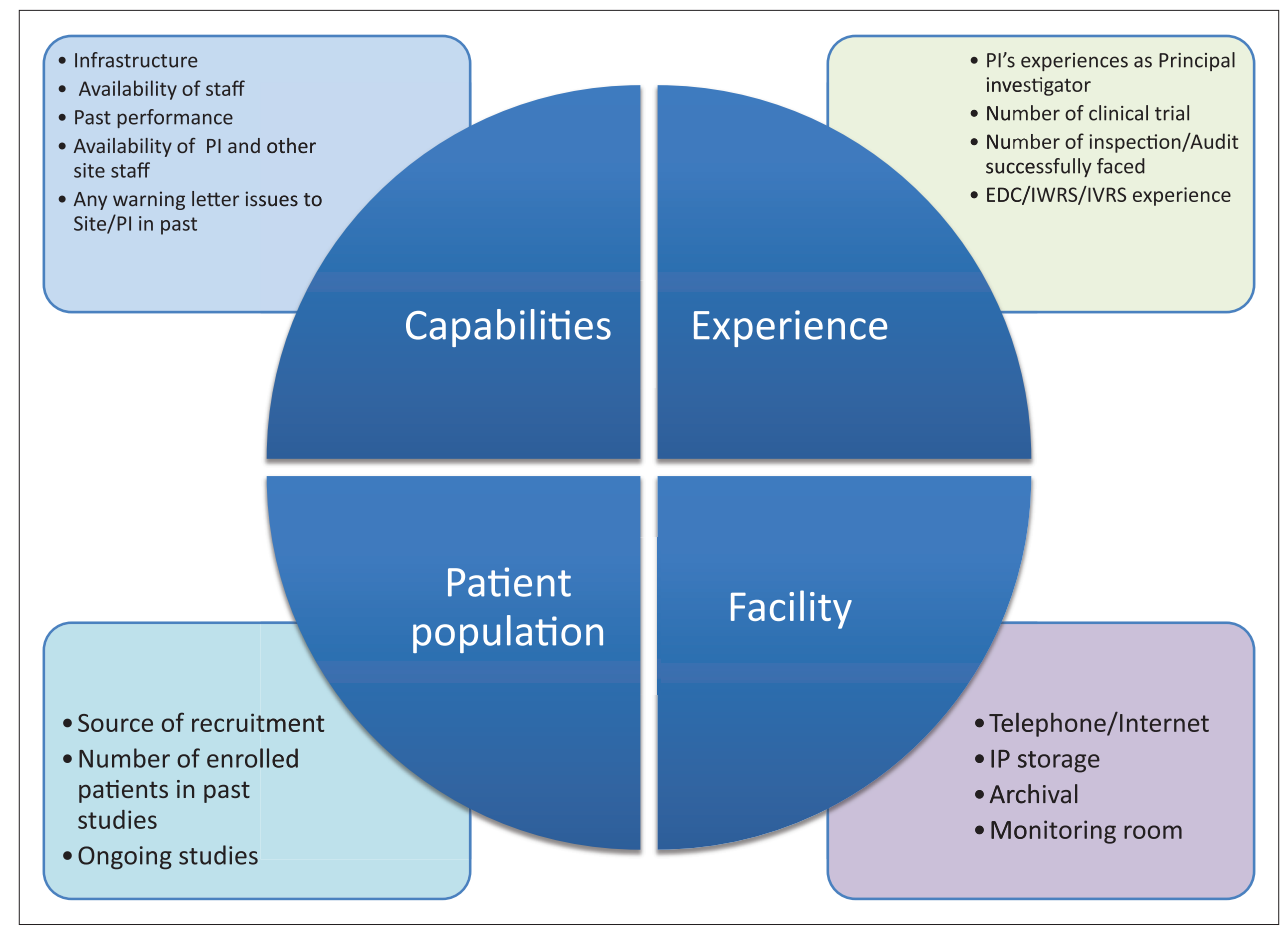

Fig. 2: Elements for site selection 
Other factors there are various other factors which also influence the cost of clinical trial. There is ethics committee cost which would increase with the cost of protocol and informed consent form amendments, administrative staff costs constitute around $11 \%-29 \%$ and can be reduced by effective resource management. Central laboratory supplements significant costs (4-12\%) [1] and number of additional procedure can increase the cost of a clinical trial by $65 \%$ [5]. Local labs are cheaper compared to central labs and using local lab may reduce the cost significantly.

It is important to note that shifting clinical trial sites to India, China, and other emerging countries may reduce the cost around 60\% [17] despite the fact that some of the related cost would increase, e.g., the cost of translation cost, especially in those regions where English is not the primary or official language and translation is done for various regulatory requirements. However, it is observed that various documents are unnecessarily translated as a precautionary measure with no use and avoiding them could help in saving some cost.

Increasing complexities and globalization of clinical trial has also lead to shipment challenges. It is easier to ship the investigational drug to one country or region as compared to the shipment to multiple countries and regions as it would require temperature control, regulatory restrictions, import license to name a few. Although data management generally does not add to the avoidable cost; however, timely integration of various software and data platform can also play a big role in delay and cost saving. Study budget negation and finalization also cause delay for $49 \%$ of the study [18].

\section{PROJECT MANAGER - A KEY STAKEHOLDER}

A clinical trial project consists of typical phases of a project, i.e. initiating planning, executing, monitoring and controlling, and analysis and reporting. It requires lots of planning and effective execution to complete the clinical trial successfully. A typical drug development project would have a project leader who works as guiding force for the several clinical trials in various phases. Project leader would have many project managers, each responsible for conduct of a clinical trial, reporting to him directly for the progress of the clinical trials. Project managers are well aware of the progress of clinical trial and any change in the timelines, cost and resourcing due to several factors. Project leader can decide the next stage of development based on the information received from other project managers. There could be cases of holding the further development for another clinical trial due to safety issues reported in one of the trial. This could result in huge cost saving. Therefore, one of the most important role of a project leader is to keep the communication live between various project managers and seek their inputs based on the progress of the trial.

Further project managers need to focus on each stage of clinical trial and manage the resource as required while getting the best and timely outcome of a clinical trial. They are expected to take the decision about site related cost, location of region and sites, keep an eye of the progress of clinical trial and safety inputs and the trend for protocol deviation. Other significant tasks of a project manager are to a track time and cost of the trial and report it project leader in a timely manner. An efficient project manager is one who keeps the ball rolling and works as an integrator for various member of the team.

\section{CONCLUSION}

Increasing drug development cost has become a challenge for pharmaceutical industry and is impacting the launch of new drug in the market. There are 5000 drugs in the development phase worldwide [4] across various therapeutic areas which reflect the money at risk as only around $10 \%$ of the drug candidate entering clinical trial would pass [19]. This can certainly be reduced by improving the processes and adapting effective project management practices in place. The money saved can be utilized by the pharmaceutical companies for future development. Reduction in cost of drug development will also encourage other mid and small size pharmaceuticals to come forward and invest in drug development.

\section{REFERENCES}

1. Sertkaya A, Birkenbach A, Berlind A, Eyraud J. Examination of Clinical Trial Costs and Barriers for Drug Development. July; 2014. Available from: http://www.aspe.hhs.gov. [Last accessed on 2016 Jun 07].

2. Kureishi A. Asia: A New Frontier in Strategic Drug Development; 2011. Available from: http://www.quintiles.com. [Last accessed on 2016 Jun 07].

2. Slater T, Bouton C, Huang ES. Beyond data integration. Drug Discov Today 2008;13(13-14):584-9.

4. Battelle Technology Partnership Practice. Biopharmaceutical Industry-Sponsored Clinical Trials: Impact on State Economies. March; 2015. Available from: http://www.phrma.org. [Last accessed on 2016 Jun 07].

5. Schultz H. Closing the Variance Gap: The Challenges with Clinical Trial Budget Management and Forecasting. May; 2015. Available from: http://www.appliedclinicaltrialsonline.com. [Last accessed on 2016 Jun 07].

6. Quintiles White Paper. Improving Clinical Development in Emerging Biopharma Settings: How Model Based Drug Development Leads to Smarter, More Predictable Trials. Available from: http://www.quintiles. com. [Last accessed on 2016 Jun 07].

7. Morrison BW, Cochranb CJ, Whitec JG, Harleyd J, Kleppingere CF, Liu A, et al. Monitoring the quality of conduct of clinical trials: A survey of current practices. SAGE J 2011. Available from: http:// www.ctj.sagepub.com.

8. Getz K. Clinical Trial Complexity. USA: Tufts CSDD, Tufts University SchoolofMedicine;2012.Availablefrom:http://www.nationalacademies. org/hmd/ /media/34D1A23404A8492998AD2DF0CB6CD4D1.ashx.

9. Johnson J. Clinical Protocols: Improve Your Designs and Reduce Costs. Feb; 2016. Available from: http://www.intilaris.com. [Last accessed on 2016Jun 07].

10. El-Assi Z. Cost forecasting for clinical trial management. Am Pharm Rev Suppl 2015;18-21. Available from: http://www.niceinsight.com/ Userfiles/Articles/PDFs/APR Supplement 0415 Merge.pdf.

11. Covance, Addressing Operational Challenges in Conducting Clinical Trials. July; 2015. Available from: http://www.covance.com. [Last accessed on 2016 Jun 07].

12. Sacks LV, Shamsuddin HH, Yasinskaya YI, Bouri K, Lanthier ML, Sherman RE. Scientific and regulatory reasons for delay and denial of FDA approval of initial applications for new drugs, 2000-2012. JAMA 2014;311(4):378-84.

13. Lyle Fitzsimmons. Clinical Trial Site Selection: Research Reality Check. January, 2010. Available from: http://www.iconplc.com. [Last accessed on 2016 Jun 07].

14. Clinipace. Trends in Clinical Trial Site Selection \& Patient Recruitment. Available from: http://www.clinipace.com. [Last accessed on 2016 Jun 07].

15. Cutting Edge Information. Clinical Trial Budgets Decreased Among Surveyed Companies by Implementing Risk-Based Monitoring Approaches, According to Cutting Edge Information. February, 2016. Available from: http://www.marketwired.com. [Last accessed on 2016 Jun 07].

16. Quintiles White Paper. Transform Clinical Development Through Advances in Risk-Based Monitoring; 2015. [Last accessed on 2016 Jun 07].

17. Bhowmik D, Chandira M, Chiranjib B. Emerging trends of scope and opportunities clinical trials In India. Int J Pharm Pharm Sci 2010;2 Supp 1:7-20.

18. Farfel G, Neuer A. Faster Study Start-Up and Reduced Costs through the Use of Clinical Document Exchange Portals; 2009. Available from: http://www.intralinks.com.

19. Umashankar V, Gurunathan S. Drug discovery: An appraisal. Int J Pharm Pharm Sci 2015;7(4):59-66. 